\title{
Damage evolution in metals under the loading due to the contact with surface-active liquid
}

\author{
O.B. Naimark ${ }^{\dagger}$, M.V. Bannikov \\ †naimark@icmm.ru
}

Institute of Continuous Media Mechanics Ural Branch of RAS, Perm, Russia

Features of the Rehbinder effect, which consist in a qualitative change of the development of multi-scale damage kinetics in a deformed sample with presence of surfactants (in the present case liquid gallium), its influence on the fatigue life of pure iron in gigacycle loading conditions are associated with qualitative changes in the role of the surface. The property of the surface to serve as a "sink" for high power for defects is significantly reduced due to the similarity of the chemical potential of the solid and surfactant as a result of "filling-in" incomplete atomic planes that provides an "adiabatic" process of accumulation of the damage in the volume of material. Fatigue tests of pure iron were carried out on ultrasonic resonant fatigue machine Shimadzu USF-2000 with frequency $20 \mathrm{kHz}$ in the so-called gigacycle fatigue regime when material failure occurs on reaching the number of loading cycles up to $10^{9}$ with very low stress amplitude. Fractured surfaces were analyzed by optical and electronic microscopes to identify the depth of penetration of liquid metal into the fracture area. It is shown that the durability and strength of the material are significantly reduced in the "adiabatic" surface, the region of localization of the defect density is shifted to the surface layer, which is characteristic for "embrittlement" materials in contact with surfactant. Rehbinder effect radically changes the mechanism of crack initiation in gigacycle fatigue regime, which leads to the formation of cracks in the surface layer of the sample, rather than the volume of the material, which is characteristic of this kind of fatigue failure.

Keywords: Rehbinder effect, defects kinetics, damage localization, adiabatic property of surface as the as a sink for defects, gigacycle fatigue.

\section{Развитие поврежденности в металлах при нагружении в условиях контакта с ПАВ}

\author{
Наймарк О.Б. ${ }^{\dagger}$, Банников М.В. \\ †naimark@icmm.ru
}

Институт механики сплошных сред УрО РАН, ул. Ак. Королева 1, 614013, Пермь, Россия

Особенности проявления эффекта Ребиндера, заключающиеся в качественном изменении многомасштабной кинетики развития поврежденности в деформируемом образце в присутствии поверхностно активных веществ (ПАВ) (в данной работе - жидкого галлия), его влияние на усталостную долговечность чистого железа при гигацикловом режиме нагружения связываются с качественным изменением роли поверхности. Свойство поверхности действовать в качестве «стока» большой мощности для дефектов оказывается существенно сниженным при близости химических потенциалов твердого тела и ПАВ вследствие «достраивания» незавершенных атомных плоскостей, что обеспечивает «адиабатичность» процесса накопления поврежденности в объеме материала. Усталостные испытания чистого железа проведены на ультразвуковой резонансной нагружающей машине Shimadzu USF-2000 с частотой испытаний 20 кГц в режиме так называемой гигацикловой усталости, когда при крайне низких амплитудах напряжения (ниже предела пропорциональности) материал разрушается при достижении количества циклов нагружения до величин $10^{9}$. Поверхность разрушения исследовалась оптическим и сканирующим электронным микроскопом с целью определения глубины проникновения жидкого металла в область разрушения. Показано, что долговечность и прочность материала существенно снижаются при контакте с ПАВ («адиабатичности» поверхности), при этом область локализации плотности дефектов смещается в приповерхностный слой, что характерно для проявлений «охрупчивания» материалов при контакте с ПАВ. Эффект Ребиндера кардинально изменяет механизм зарождения трещины при гигацикловом режиме усталости, что приводит к образованию трещины в приповерхностном слое образца, а не в объеме материала, что характерно для режима гигациклового нагружения.

Ключевые слова: Эффект Ребиндера, кинетика дефектов, локализация поврежденности, адиабатичность поверхности «как стока» дефектов, гигацикловая усталость. 


\section{1. Введение}

Механические свойства (прочность, пластичность) твердых тел различной физической природы могут существенно изменяться под влиянием окружающей среды. В целом эти изменения разнообразны и могут быть связаны как с химическим взаимодействием, так и с физико-химическим влиянием среды. Во втором случае изменения свойств твердых тел, известные как эффект Ребиндера, связываются обычно с понижением удельной поверхностной энергии тела, в том числе и работы образования его новых поверхностей (трещин) в момент их возникновения. Отличительные особенности этого эффекта заключаются в том, что они наблюдаются только при совместном действии поверхностно-активной среды и напряженного состояния. Степень проявления эффекта зависит от химического состава твердого тела и окружающей среды, от структуры материала и условий деформирования [1]. Значительное уменьшение разрывных напряжений и деформаций (как в экспериментах Ребиндера - деформирование цинкового уса в ртути) свидетельствует о качественном изменении условий деформирования.

Исследования закономерностей эффекта Ребиндера при различных скоростях деформирования указывают на чисто поверхностное влияние окружающей среды, с пренебрежимо малой глубиной диффузии атомов ПАВ в объем материала за время разрушения. Особенности проявления эффекта Ребиндера дают основания предположить, что деформируемый образец должен рассматриваться в общем случае как часть системы «твердое тело - ПАВ». Если же математическое описание процесса ограничено объемом образца, то влияние окружающей среды должно быть отражено в граничных условиях. Применительно к краевой задаче разрушения речь идет об определении граничных условий для параметра поврежденности, ассоциируемого с реальной дефектной структурой материала, в условиях контакта на поверхности образца с ПАВ, которые могут существенно влиять на кинетику развития дефектов, его стадийность, локализацию поврежденности.

\section{2. Феноменология твердых тел с дефектами}

Структурные переменные, ассоциированные с типичными дефектами (дислокационными скоплениями, микротрещинами и микросдвигами), были введены в [2] и описываются макроскопическим тензором плотности дефектов $p_{i k}$, который совпадает по смыслу с деформацией, обусловленной дефектами. Статистическое описание позволило предложить феноменологию твердого тела с дефектами, основанную на представлении о неравновесной свободной энергии $F$, отражающем качественное изменение нелинейного поведения ансамбля дефектов на различных стадиях поврежденности. Феноменологическое представление части свободной энергии, обусловленной ростом плотности дефектов [2] в поле внешних напряжений $\sigma_{i k}$, дается шестым порядком разложения, аналогичного хорошо известному раз- ложению Гинзбурга-Ландау [3]

$$
\begin{aligned}
& \mathrm{F}=1 / 2 \mathrm{~A}\left(1-\delta / \delta_{*}\right) \mathrm{p}_{\mathrm{ik}}^{2}-1 / 4 \mathrm{Bp}_{\mathrm{ik}}^{4}+ \\
& +1 / 6 \mathrm{C}\left(1-\delta / \delta_{\mathrm{c}}\right) \mathrm{p}_{\mathrm{ik}}^{6}-\mathrm{D} \sigma_{\mathrm{ik}} \mathrm{p}_{\mathrm{ik}}+\chi\left(\nabla_{1} \mathrm{p}_{\mathrm{ik}}\right)^{2},
\end{aligned}
$$

где $\delta$ - параметр структурного скейлинга, представляющий собой отношение двух характерных пространственных масштабов для среды с дефектами: расстояние между дефектами и размер дефекта. Градиентный член в (1) описывает эффекты нелокальности в ансамбле дефектов; $A, B, C, D$ и $\chi$ - феноменологические параметры. Параметр структурного скейлинга является независимой переменной для среды с дефектами и определяет текущую восприимчивость материала к росту дефектов. Критические значения $\delta_{*}, \delta_{c}$ разделяют характерные типы нелинейностей для среды с дефектами и играют роль, аналогичную характеристическим температурам в теории фазовых переходов Ландау. Этот факт имеет существенное значение при обобщении термодинамических соотношений применительно к поведению систем, далеких о равновесия [3]. Кинетическое уравнение для параметра плотности дефектов, в дальнейшем рассматривается случай одноосного деформирования $\sigma_{i k} \sim \sigma_{z z}=\sigma$, для которого тензор плотности дефектов имеет компоненту $p_{z z}=p$, следует из эволюционного неравенства $\delta F / \delta t=\delta F / \delta p \cdot d p / d t \leq 0[3]$

$$
\begin{aligned}
\frac{\mathrm{dp}}{\mathrm{dt}}=-\Gamma\left(\mathrm{A}\left(1-\frac{\delta}{\delta}\right) \mathrm{p}-\mathrm{Bp}^{3}+\right. & \\
& \left.+\mathrm{C}\left(1-\frac{\delta}{\delta_{\mathrm{c}}}\right) \mathrm{p}^{5}-\mathrm{D} \sigma-\frac{\partial}{\partial \mathrm{x}_{1}}\left(\chi \frac{\partial \mathrm{p}}{\partial \mathrm{x}_{1}}\right)\right)
\end{aligned}
$$

где Г - кинетический коэффициент.

Данное уравнение имеет различные групповые свойства в соответствующих диапазонах параметра структурного скейлинга $\delta$, определяемые видом нелинейности неравновесного потенциала $F$, включая тип метастабильности. При $\delta \rightarrow \delta_{*}$ происходит вырождение решений, характерных для параболического уравнения с нелинейным источником, и последнее трансформируется в волновое решение автосолитонного типа $p(\zeta)=p(x-V t)$ для бистабильного потенциала. Амплитуда волны, её ширина и скорость волнового фронта определяются параметрами метастабильного перехода

$$
\begin{gathered}
\mathrm{p}=\frac{1}{2} \mathrm{p}_{\mathrm{a}}\left[1-\tanh \left(\zeta 1^{-1}\right)\right], \quad 1=\frac{4}{\mathrm{p}_{\mathrm{a}}}\left(2 \frac{\chi}{\mathrm{A}}\right)^{1 / 2}, \\
\mathrm{~V}=\chi \mathrm{A}\left(\mathrm{p}_{\mathrm{a}}-\mathrm{p}_{\mathrm{m}}\right) / 2 \zeta^{2},
\end{gathered}
$$

где $\left(p_{a}-p_{m}\right)-$ скачок величины $p$ в ходе метастабильного перехода. Формирование пространственно локализованных волновых структур, фронты которых соответствуют «ориентационному переходу» в ансамблях дефектов, позволяют связать кинетику формирования этих структур с механизмами пластической деформации.

Переход через точку бифуркации $\delta_{c}$ сопровождается очередным изменением групповых свойств уравнения (2), что приводит к появлению пространственно-временных структур качественно нового типа, описывающих «взрывное» накопление дефектов (так называемые режимы с обострением [3]) при $t \rightarrow t_{c}$ на спектре про- 
странственных масштабов $L_{H}=k L_{c}, k=1,2, \ldots K$. Для данного типа уравнений, как показано в [4], развитая стадия кинетики $p>p_{c}$ при $t \rightarrow t_{c}$ описывается автомодельным решением

$$
\mathrm{p}(\mathrm{x}, \mathrm{t})=\varphi(\mathrm{t}) \mathrm{f}(\zeta), \quad \zeta=\frac{\mathrm{x}}{\mathrm{L}_{\mathrm{c}}}, \quad \varphi(\mathrm{t})=\Phi_{0}\left(1-\frac{\mathrm{t}}{\mathrm{t}_{\mathrm{c}}}\right)^{-\mathrm{m}},
$$

где $m>0, \Phi_{0}>0-$ параметры, определяемые показателями нелинейности потенциала $(1) ; L_{c}$ и $t_{c}$ - параметры скейлинга. Функция $f(\zeta)$ может быть определена в ходе решения нелинейной задачи на собственные значения [4]. “Подчинение” кинетики роста дефектов режиму с обострением соответствует сценариям автомодельности второго рода с вырождением масштабов, сформированных коллективными автосолитонными модами, но уже в нерегулярном (асимптотическом) режиме. Формирование структур обострения обеспечивает наиболее эффективный канал диссипации, который может быть связан в сплошной среде с зарождением очагов макроскопического разрушения (трещин) в твердых телах.

\section{3. Роль поверхности в развитии дефектов}

Поверхность материала, как отмечал Френкель [5], играет особую роль в процессе необратимой деформации твердых тел, являясь источником и стоком большой мощности для дефектов. Поскольку необратимая деформация определяется нелокальными взаимодействиями, диффузионными процессами, в основе которых лежит взаимодействие дислокаций между собой и с вакансиями, в [6] было высказано предположение, что ПАВ может сильно влиять на диффузионные процессы при взаимодействии, например, дислокационных петель с поверхностью твердого тела. При этом выход дислокаций на внешнюю границу завершает микроскопический акт необратимого деформирования. Поэтому отсутствие или недостаточная мощность внешнего стока для дислокаций (свободной поверхности) приводит к рассогласованию процессов их зарождения и движения в макроскопическом объеме, что влияет на развитие пластичности, упрочнения и локализации поврежденности, связанные с многомасштабными процессами эволюции дислокационной подсистемы.

Для параметра плотности дефектов $p_{z z}=p$, учитывая дифференциальный порядок эволюционного уравнения (2), можно записать следующий вид граничных условий на поверхности $S$

$$
\frac{\partial \mathrm{p}}{\partial \mathrm{x}}=-\mathrm{Np}, \mathrm{x} \in \mathrm{S},
$$

где $N$ - константа взаимодействия, зависящая от химических потенциалов твердого тела и окружающей среды.

Влияние величины параметра взаимодействия $N$ на изменение критических режимов показало (для случая активного нагружения, деформирования с постоянной скоростью), что случай больших $N$ соответствует свойству свободной поверхности как «стока» неограниченной мощности для дефектов $\left(p_{i k}=0\right)$. При $N=0$ кинетика развития разрушения (переход к режимам обострения) реализуется за более короткое время при меньших величинах деформации и напряжений разрушения.

На основе проведенного в [7] численного анализа сделан вывод, что физико-химическое влияние окружающей среды может быть интерпретировано как эффективное запирание» свободной поверхности для движущихся дислокаций.

\section{4. Эволюция структуры деформируемого металла при контакте с ПАВ}

Действие поверхностно-активных веществ приводит к облегчению пластической деформации металлов и адсорбционному снижению предела прочности. В [6] показано, что возникающее при действии адсорбционных слоев снижение предела текучести и коэффициента упрочнения сопровождается значительным измельчением полос скольжения и зеренной структуры деформируемого металла.

При длительных циклических нагрузках развитие дефектной структуры концентрируется, как правило, в поверхностных слоях металла. Установлено [6,7], что при контакте поверхности деформируемого металла с поверхностно-активной средой, возникновение дефектов структуры облегчается, что сопровождается ростом поверхностной разрыхленности металла. В [8] с использованием данных электронной микроскопии показано, что при статическом растяжении стали в присутствии поверхностно-активной среды происходят значительные изменения в дислокационной структуре по сравнению со структурой, возникающей при деформации в неактивной среде. Исследование особенностей формирования дислокационной структуры стали в условиях усталости показало, что при одинаковых режимах нагружения стальных образцов в инертной и в поверхностноактивной среде наблюдается снижение выносливости до 30-50\% в случае контакта с поверхностно-активной средой. Усталостное нагружение в присутствии ПАВ приводит к значительным изменениям в дислокационной структуре по сравнению со структурой, формирующейся при той же нагрузке в активной среде. Установлено, что испытание в поверхностно-активной среде приводит к увеличению плотности дислокаций с более сложным взаимодействием в ансамбле дислокаций. Оценка плотности дислокаций по данным электронной микроскопии показала, что при нагружении в присутствии ПАВ плотность дислокаций возрастает в 3-5 раз. Наблюдаемая более высокая плотность дислокаций в поверхностных слоях при нагружении в поверхностно-активной среде свидетельствует о том, что в этих условиях протекание пластической деформации облегчено. Повышение активности подповерхностных источников приводит к дополнительному увеличению плотности дислокаций и их взаимодействию. Следствием этого является формирование грубой дислокационной сетки в условиях более интенсивного протекания упрочнения при усталости. В результате способность металла к упрочнению исчерпывается при меньшем числе циклов, и последующие стадии усталости и, как следствие, разрушение наступают 
раньше.

Наблюдаемое формирование мозаичной структуры кристаллов при образовании дислокационных стенок высокой плотности является следствием повышения плотности дислокаций в поверхностных слоях в условиях воздействия адсорбционных сред, что приводит к интенсивному измельчению кристаллитов и блоков.

\section{5. Материал и условия эксперимента}

В работе исследуется эффект адсорбционного понижения прочности чистого железа под действием жидкого эвтектоидного сплава на основе галлия с оловом и индием в режиме гигацикловой усталости. Данный вид испытаний позволяет установить качественные изменения в стадийности развития поврежденности в связи с типичным для условий гигациклового нагружения формированием очага макроскопического разрушения («fish-eye area») в объеме образца. Испытания проводились на ультразвуковой нагружающей машине резонансного типа Shimadzu USF-2000. На Рис. 1 представлена принципиальная схема установки. Пьезоэлектрический преобразователь (конвертер) трансформирует электрические колебания ультразвукового генератора в механические периодические воздействия с частотой 20 КГц. Установка обеспечивает циклическое нагружение образца в режиме ультразвукового резонанса. Амплитуда смещения достигает максимума $\mathrm{U}_{0}$ на свободном торце образца и измеряется динамическим сенсором. Максимальное напряжение сосредоточено в центре образца. Распределение смещений и напряжений в системе конвертер-усилитель-образец с коэффициентом асимметрии цикла $\mathrm{R}=-1$ изображено на рис. 1 . В течение ультразвукового усталостного испытания деформация может быть напрямую измерена с помощью тензометрических датчиков, закрепленных на поверхности образца. Номинальная резонансная частота вибраций нагружающего устройства близка к 20 кГц и нарушение условий резонанса более чем 0.5 кГц является критерием остановки испытания, что связывается с образованием усталостной трещины. Таким образом, если система контроля обнаружит частоту менее 19.5 кГц, то тест будет остановлен.

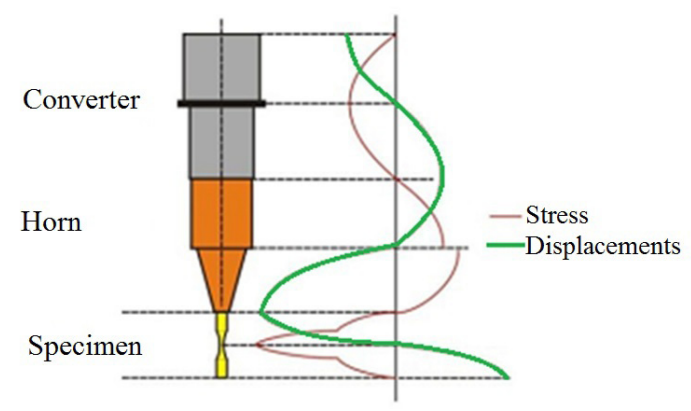

Рис. 1. Поля напряжений и смещений системы конвертерусилитель-образец в резонансной нагружающей машине.

Fig. 1. Stress and displacements diagrams in converter-hornspecimen system in ultrasonic fatigue machine.

\section{6. Результаты усталостных испытаний}

На рис. 2 представлены результаты испытания для образцов армко-железа на воздухе и при контакте с жидким металлом. Анализ данных [9] позволяют сделать вывод о том, что для рассматриваемого материала на воздухе существует предполагаемый предел усталости на базе испытаний $3 \cdot 10^{9}$ при напряжении 160 МПа с типичным сценарием зарождения трещины («fish-еуе») в объеме образца. Однако при нанесении тонкого слоя жидкого галлия на поверхность образца, его усталостная долговечность снижается более чем на 2 порядка (6·10 $-2,3 \cdot 10^{7}$ циклов до разрушения).

Потеря образцом резонансной частоты на ультразвуковой машине является следствием нелинейных процессов, обусловленных изменением упругих свойств и эффектами неупругости, предшествующих зарождению усталостной трещины. В случае железа потеря резонансной частоты и, как следствие, остановка нагружения не всегда означала механическое (макроскопическое) разрушение образца с образованием трещины. Такой характер поведения образцов является следствием необратимых изменений в структуре материала, которые можно считать критическими с точки зрения деформационного ресурса образца при ультразвуковом нагружении. При этом изменения не связаны с образованием усталостной трещины и механически соответствуют резкому повышению диссипативной способности образца и невозможности его испытаний с воздушным охлаждением, предусмотренным на усталостной машине. Измерения температуры образца в процессе испытаний показали, что на завершающей стадии процесса наблюдается резкий рост температуры образца до $220^{\circ} \mathrm{C}$, сопровождающийся падением резонансной частоты [10].

В случае ультразвукового нагружения образца в присутствии жидкого галлия (рис. 3) обнаруживалась усталостная трещина, зародившаяся с поверхности образца как при механизмах классической многоцикловой усталости. Края трещины свидетельствовали о проникновении жидкого металла в трещину в процессе усталостных испытаний.

Определяющую роль в развитии разрушения при гигацикловой усталости играет стадия зарождения усталостной трещины, а процесс её распространения в материале занимает 2-5\% от долговечности материала $[9,11]$. Тот факт, что при одинаковом уровне напряжения усталостное разрушение происходит при меньшем на несколько порядков количестве циклов, свидетельствует об изменении механизма зарождения трещины под влиянием поверхностно активного вещества.

«Критическое сечение» образцов «вскрывалось» в условиях предварительного охлаждения образца с помощью жидкого азота и исследовалось оптическим профилометром и электронным сканирующим микроскопом (рис.3). Анализ фрактографии изломов позволил установить, что трещина в присутствии жидкого металла зарождается в приповерхностном слое материала. Анализ химических элементов, проведенный по отраженным от поверхности разрушения спектрам в сканирующем электронном микроскопе, позволил определить, что жидкий 
металл проникает внутрь материала на глубину около 200 мкм и находится исключительно в очаге зарождения трещины.

Таким образом можно говорить о том, что эффект жидкометаллического охрупчивания проявляется преимущественно на стадии образования усталостной трещины, а ее продвижение внутрь происходит по классическому закону Пэриса. Об этом факте также могут свидетельствовать результаты, полученные в [8] для мало- и многоцикловой усталости, когда снижение долговечности наблюдалось в несколько раз, так как стадия зарождения усталостной трещины в режимах мало- и многоцикловой усталости значительно менее продолжительна.

\section{7. Заключение}

Учитывая особенность проявления эффекта Ребиндера, заключающуюся в качественном изменении многомасштабной кинетики развития дислокационной подсистемы в приповерхностном слое деформируемого образца в присутствие ПАВ, влияние поверхностно-активной среды (жидкого металла) на усталостную долговечность чистого железа в гигацикловом режиме нагружения связывается с качественным изменением роли поверхности. Свойство поверхности действовать как "сток» большой мощности для дефектов оказывается суще-

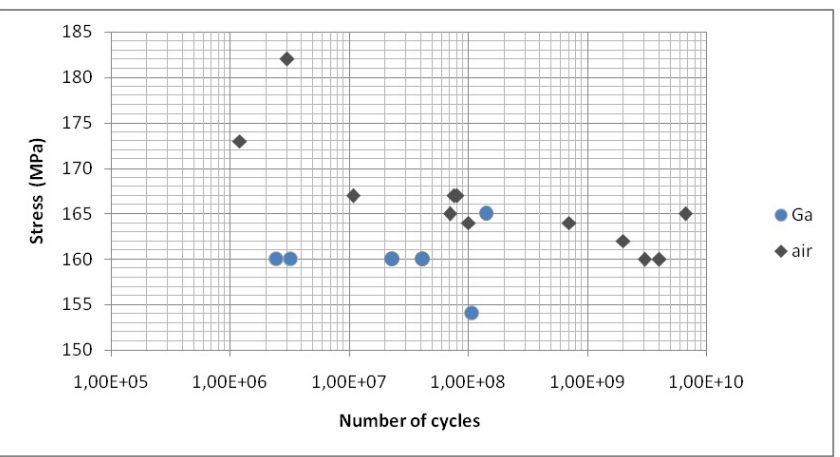

Рис. 2. Результаты усталостных испытаний армко-железа на воздухе и при контакте с жидким металлом.

Fig. 2. Results of fatigue tests of pure iron on air and in contact with liquid gallium.

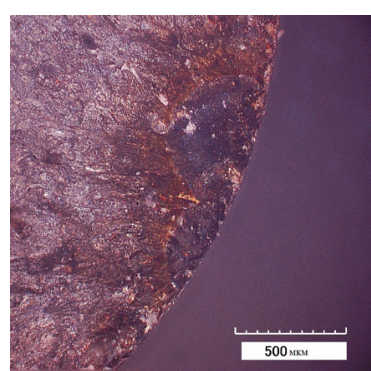

a)

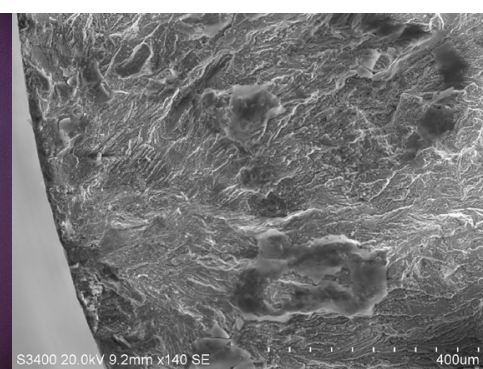

b)
Рис. 3. Очаг зарождения усталостной трещины а) оптическая микроскопия; b) электронная микроскопия.

Fig. 3. Fatigue crack origin a) optical microscopy; b) electronic microscopy. ственно сниженным при близости химических потенциалов твердого тела и ПАВ вследствие «достраивания» незавершенных атомных плоскостей, что обеспечивает «адиабатичность» процесса накопления поврежденности в объеме материала. Исследование кинетики поврежденности при деформировании материала в зависимости от величины параметра $N$ представлено в [7]. Показано, что долговечность и прочность материала снижаются существенно при уменьшении параметра $N$, при этом область локализации плотности дефектов смещается в приповерхностный слой, что характерно для проявлений «охрупчивания» материалов при контакте с ПАВ. Эффект Ребиндера кардинально изменяет механизм зарождения трещины в гигацикловом режиме усталости, что приводит к образованию трещины в приповерхностном слое образца, а не в объеме материала, что характерно для режима гигациклового нагружения.

Благодарность. Работа выполнена при поддержке гранта РНФ 14-19-01173.

Acknowledgements. This study was supported by the Russian Science Foundation, project No. 14-19-01173.

\section{Литература/References}

1. P.A. Rehbinder, E.D. Shchukin, Physics-Uspekhi (1972) 108, 1,(in Russian) [Ребиндер П.А., Щукин Е.Д. // Усп. физ. наук. 1972. 108.C. 1. ]

2. O.B. Naimark / Phys. Mesomech. Jour. (2003), V.4.- P.4572. (In Russian) [Наймарк О.Б. / Физ. Мезомех.- 2003. - T.4.- C.45-72.]

3. Naimark O.B. / In: Advances in multifield theories of continua with substructure. Ed.: G.Capriz, P.Mariano.Boston, Birkhauser.- 2004. -P.75-114.

4. O.B. Naimark / Phys. Mesomech. Jour. (2010), V.13(5).P.113-126 (In Russian) [Наймарк, О.Б. /Физ. Мезомех. - 2010. - T.13. - №5. - C.113-126.]

5. Ya.I. Frenkel, Kineticheskaya teoriya zhidkosti. Leningrad: Nauka (1975) P.592 (in Russian) [ Я.И. Френкель, Кинетическая теория жидкости.Ленинград: Наука.- 1975.-С.592

6. Naimark O.B., Silberschmidt V.V. / Eur.J. of Mechanics, 1991, n.6, p.607-619.

7. O. B. Naimark, O. A. Plekhov, V. I. Betekhtin, A. G. Kadomtsev, M. V. Narykova / Technical Physics (2014), V 59, Iss 3, pp 398-401

8. V.V. Shevelya, B.I. Kosteckiy, Doklady Physics, (1967) ,175(6), p. 1270-1272, (in Russian) [Шевеля В.В., Костецкий Б.И. / Доклады Академии Наук, 1967, т. 175, №6, 1270-1272]

9. C. Bathias, P. C. Paris. Gigacycle Fatigue in Mechanical Practice . Marcel, Dekker Publisher Co. (2005) 328 p.

10. A.N. Vshivkov, A.E. Prokhorov, S.V.Uvarov, O.A. Plekhov // PNRPU Mechanics Bulletin, 4,18-32,( 2013). [A.H. Вшивков, А.Е. Прохоров, С.В. Уваров, О.А. Плехов // Вестник ПНИПУ Механика, 2013, № 4, с. 18-32.]

11. Mughrabi H. / Int J Fatigue. 2013, Vol. 57, pp. 2-8. 